\title{
Neuronavigated repetitive transcranial magnetic stimulation as novel mapping technique provides insights into language function in primary progressive aphasia
}

\author{
Felix Mueller-Sarnowski ${ }^{1,2} \cdot$ Nico Sollmann $^{3,4,5} \cdot$ Axel Schröder $^{6} \cdot$ Leen Houri $^{1} \cdot$ Sebastian Ille $^{6}$ - Timo Grimmer ${ }^{1}$. \\ Sandro M. Krieg ${ }^{4,6} \cdot$ Janine Diehl-Schmid ${ }^{1,7}$ iD
}

Accepted: 15 November 2021 / Published online: 28 December 2021

(c) The Author(s) 2021

\begin{abstract}
Navigated repetitive transcranial magnetic stimulation (nrTMS) is an innovative technique that provides insight into language function with high accuracy in time and space. So far, nrTMS has mainly been applied in presurgical language mapping of patients with intracranial neoplasms. For the present study, nrTMS was used for language mapping in primary progressive aphasia (PPA). Seven patients (median age: 70 years, 4 males) with the non-fluent variant of PPA (nfvPPA) were included in this pilot study. Trains of nrTMS (5 Hz, 100\% resting motor threshold) caused virtual lesions at 46 standardized cortical stimulation targets per hemisphere. Patients' errors in a naming task during stimulation were counted. The majority of errors induced occurred during frontal lobe stimulation (34.3\%). Timing errors and non-responses were most frequent. More errors were induced in the right hemisphere (58\%) than in the left hemisphere (42\%). Mapping was tolerated by all patients, however, discomfort or pain was reported for stimulation of frontal areas. The elevated right-hemispheric error rate in our study could be due to a partial shift of language function to the right hemisphere in neurodegenerative aphasia during the course of disease and therefore points to the existence of neuronal plasticity in nfvPPA. While this is an interesting finding for neurodegenerative disorders per se, its promotion might also harbor future therapeutic targets.
\end{abstract}

Keywords Navigated repetitive transcranial magnetic stimulation · Primary progressive aphasia $\cdot$ Language mapping · Cortical plasticity

Janine Diehl-Schmid

janine.diehl-schmid@tum.de

1 Department of Psychiatry and Psychotherapy, School of Medicine, Klinikum Rechts der Isar, Technical University of Munich, Ismaninger Str. 22, 81675 Munich, Germany

2 Department of Medical Information Science, School of Medicine, Augsburg University, Augsburg, Germany

3 Department of Diagnostic and Interventional Neuroradiology, School of Medicine, Klinikum Rechts der Isar, Technical University of Munich, Munich, Germany

4 TUM-Neuroimaging Center, Klinikum Rechts der Isar, Technical University of Munich, Munich, Germany

5 Department of Diagnostic and Interventional Radiology, University Hospital Ulm, Ulm, Germany

6 Department of Neurosurgery, School of Medicine, Klinikum Rechts der Isar, Technical University of Munich, Munich, Germany

7 Munich Cluster for Systems Neurology (SyNergy), Munich, Germany

\section{Introduction}

Primary progressive aphasias (PPAs) are gradually progressive, neurodegenerative disorders predominantly affecting speech and language. The non-fluent variant of PPA (nfvPPA) is characterized by a predominant posterior frontoinsular atrophy of the language-dominant hemisphere. Patients present with a non-fluent, effortful, halting speech that is dominated by obvious word-finding difficulties and agrammatism. Language production is simplified, phonematic paraphasias are frequent. Patients often present with apraxia of speech while dysarthria occurs less frequently (Gorno-Tempini et al., 2011).

Therapeutic options are scarce and consist mainly of speech therapeutic approaches. A few studies have made first, not very successful attempts to improve language function using repetitive transcranial magnetic stimulation (rTMS) and transcranial direct current stimulation (tDCS) (Norise \& Hamilton, 2017; Cotelli et al., 2012, 2020). 
However, targeted regions and stimulation parameters vary widely in these attempts, including anodal tDCS targeting of the left inferior frontal gyrus, high-frequency rTMS of the whole left hemisphere, high-frequency rTMS of the left prefrontal cortex, or anodal (facilitative) stimulation of the left posterior peri-Sylvian region. This variability is the consequence of the sparse rationale for such interventions.

Knowledge about language function and compensatory mechanisms in the individual patient is a prerequisite for a more personalized and thus more efficient neurostimulation in nfvPPA. Positron emission tomography (PET) and functional magnetic resonance imaging (MRI) during a language task could generate such information, but only with limited resolution in time. Navigated rTMS (nrTMS) has been shown to be a reliable technique in order to perform presurgical language mapping in patients with intracranial neoplasms (Krieg et al., 2014; Picht et al., 2013; Sollmann et al., 2017b; Tarapore et al., 2013). Therefore, the primary aim of our pilot study was to investigate, which brain regions are involved in tasks of language and speech in patients with nfvPPA using nrTMS. Secondly, we wanted to investigate, how patients with nfvPPA tolerate nrTMS.

\section{Methods}

\section{Patients}

Patients with nfvPPA according to the Gorno-Tempini criteria (Gorno-Tempini et al., 2011) were enrolled from our outpatient clinic for cognitive disorders. Patient characterization comprised neurologic and psychiatric examination, and extensive neuropsychological testing. (Table 1).

Table.1 Patient characteristics

\begin{tabular}{|c|c|c|c|c|c|c|c|}
\hline Patient & $\# 1$ & $\# 2$ & $\# 3$ & $\# 4$ & $\# 5$ & \#6 & \#7 \\
\hline sex & male & female & male & male & female & male & female \\
\hline age & 73 & 70 & 56 & 68 & 75 & 55 & 74 \\
\hline age at onset & 70 & 66 & 54 & 67 & 72 & 52 & 71 \\
\hline education (years) & 15 & 12 & 15 & 20 & 18 & 19 & 12 \\
\hline handedness* & right & $\begin{array}{l}\text { left mixed (forced } \\
\text { right) }\end{array}$ & left (forced right) & right & right, mixed & right & right \\
\hline psychotropic drugs & - & mirtazapine $7.5 \mathrm{mg}$ & - & $\begin{array}{l}\text { escitalopram } 5 \mathrm{mg} \\
\text { quetiapine } 100 \mathrm{mg}\end{array}$ & citalopram $20 \mathrm{mg}$ & $\begin{array}{l}\text { amitriptyline } \\
5 \mathrm{mg}\end{array}$ & - \\
\hline \multirow[t]{2}{*}{ FTLD-CDR } & 3 & 1 & 0.5 & 3.5 & 2 & 5 & 5.5 \\
\hline & 0.5 & 0.5 & 0.5 & 0.5 & 0.5 & 2 & 2 \\
\hline $\begin{array}{l}\text { semantic fluency } \\
\text { (animals) }\end{array}$ & $\begin{array}{l}7 \\
(-2.8 z)\end{array}$ & $\begin{array}{l}9 \\
(-2.4 \mathrm{z})\end{array}$ & $\begin{array}{l}16 \\
(-1.6 z)\end{array}$ & $\begin{array}{l}18 \\
(-1.2 \mathrm{z})\end{array}$ & $\begin{array}{l}15 \\
(-2.2 \mathrm{z})\end{array}$ & $\begin{array}{l}2 \\
(-4.5 z)\end{array}$ & $\begin{array}{l}6 \\
(-2.9 \mathrm{z})\end{array}$ \\
\hline $\begin{array}{l}\text { phonematic fluency } \\
\text { (S-words) }\end{array}$ & $\begin{array}{l}4 \\
(-2.2 z)\end{array}$ & $\begin{array}{l}7 \\
(-1 . z)\end{array}$ & $\begin{array}{l}10 \\
(-1.0 \mathrm{z})\end{array}$ & $\begin{array}{l}5 \\
(-2.6 z)\end{array}$ & $\begin{array}{l}7 \\
(-1.9 z)\end{array}$ & $\begin{array}{l}3 \\
(-3.3 z)\end{array}$ & $\begin{array}{l}1 \\
(-3.3 \mathrm{z})\end{array}$ \\
\hline $\begin{array}{l}15 \text { item BNT } \\
(/ 15)\end{array}$ & $\begin{array}{l}14 \\
(-0.4 z)\end{array}$ & $\begin{array}{l}15 \\
(1.3 \mathrm{z})\end{array}$ & $\begin{array}{l}15 \\
(0.5 \mathrm{z})\end{array}$ & $\begin{array}{l}15 \\
(0.5 \mathrm{z})\end{array}$ & $\begin{array}{l}15 \\
(1.0 \mathrm{z})\end{array}$ & $\begin{array}{l}4 \\
(-5.9 z)\end{array}$ & $\begin{array}{l}5 \\
(-4.4 \mathrm{z})\end{array}$ \\
\hline $\begin{array}{l}\text { MMSE } \\
(/ 30)\end{array}$ & $\begin{array}{l}24 \\
(-3.1 \mathrm{z})\end{array}$ & $\begin{array}{l}29 \\
(-0.2 z)\end{array}$ & $\begin{array}{l}29 \\
(-0.8 \mathrm{z})\end{array}$ & $\begin{array}{l}29 \\
(-0.7 \mathrm{z})\end{array}$ & $\begin{array}{l}29 \\
(-0.6 z)\end{array}$ & $\begin{array}{l}20 \\
(-5.7 \mathrm{z})\end{array}$ & $\begin{array}{l}17 \\
(-6.0 \mathrm{z})\end{array}$ \\
\hline $\begin{array}{l}\text { CERAD word list learning } \\
(/ 30)\end{array}$ & $\begin{array}{l}12 \\
(-2.3 \mathrm{z})\end{array}$ & $\begin{array}{l}21 \\
(-0.3 \mathrm{z})\end{array}$ & $\begin{array}{l}24 \\
(0.6 \mathrm{z})\end{array}$ & $\begin{array}{l}24 \\
(0.9 \mathrm{z})\end{array}$ & $\begin{array}{l}19 \\
(-1.2 \mathrm{z})\end{array}$ & $\begin{array}{l}15 \\
(-2.2 \mathrm{z})\end{array}$ & $\begin{array}{l}16 \\
(-1.8 \mathrm{z})\end{array}$ \\
\hline $\begin{array}{l}\text { CERAD word list delayed recall } \\
(/ 10)\end{array}$ & $\begin{array}{l}4 \\
(-1.2 \mathrm{z})\end{array}$ & $\begin{array}{l}8 \\
(0.1 \mathrm{z})\end{array}$ & $\begin{array}{l}8 \\
(0 \mathrm{z})\end{array}$ & $\begin{array}{l}7 \\
(-0.4 \mathrm{z})\end{array}$ & $\begin{array}{l}7 \\
(-0.8 \mathrm{z})\end{array}$ & $\begin{array}{l}2 \\
(-3.0 \mathrm{z})\end{array}$ & $\begin{array}{l}5 \\
(-1.3 \mathrm{z})\end{array}$ \\
\hline $\begin{array}{l}\text { CERAD figure copy } \\
(/ 11)\end{array}$ & $\begin{array}{l}7 \\
(-2.9 \mathrm{z})\end{array}$ & $\begin{array}{l}9 \\
(-1.3 \mathrm{z})\end{array}$ & $\begin{array}{l}11 \\
(0.5 \mathrm{z})\end{array}$ & $\begin{array}{l}10 \\
(-1.4 \mathrm{z})\end{array}$ & $\begin{array}{l}11 \\
(0.6 \mathrm{z})\end{array}$ & $\begin{array}{l}11 \\
(0.3 \mathrm{z})\end{array}$ & $\begin{array}{l}10 \\
(0.5 \mathrm{z})\end{array}$ \\
\hline $\begin{array}{l}\text { CERAD figure recall } \\
(/ 11)\end{array}$ & $\begin{array}{l}4 \\
(-2.5 z)\end{array}$ & $\begin{array}{l}7 \\
(-0.9 z)\end{array}$ & $\begin{array}{l}11 \\
(0.8 \mathrm{z})\end{array}$ & $\begin{array}{l}9 \\
(-1.0 \mathrm{z})\end{array}$ & $\begin{array}{l}11 \\
(1.0 \mathrm{z})\end{array}$ & $\begin{array}{l}11 \\
(0.7 \mathrm{z})\end{array}$ & $\begin{array}{l}5 \\
(-1.5 \mathrm{z})\end{array}$ \\
\hline trail making A (seconds) & $>180$ & $\begin{array}{l}82 \\
(-1.8 z)\end{array}$ & $\begin{array}{l}46 \\
(-0.9 z)\end{array}$ & $\begin{array}{l}70 \\
(-1.8 \mathrm{z})\end{array}$ & $\begin{array}{l}65 \\
(-1.2 \mathrm{z})\end{array}$ & $\begin{array}{l}45 \\
(-0.7 \mathrm{z})\end{array}$ & $\begin{array}{l}43 \\
(0.3 \mathrm{z})\end{array}$ \\
\hline trail making B (seconds) & $\begin{array}{l}>300 \\
(-2.5 \mathrm{z})\end{array}$ & $\begin{array}{l}>300 \\
(-2.5 \mathrm{z})\end{array}$ & $\begin{array}{l}138 \\
(-1.7 \mathrm{z})\end{array}$ & $\begin{array}{l}283 \\
(-3.3 z)\end{array}$ & $\begin{array}{l}194 \\
(-2.2 \mathrm{z})\end{array}$ & $\begin{array}{l}180 \\
(-2.6 z)\end{array}$ & $\begin{array}{l}121 \\
(-0.2 \mathrm{z})\end{array}$ \\
\hline AAT token test & 5 & 12 & 12 & 1 & 3 & 42 & 5 \\
\hline AAT token test age adjusted & 0 & 6 & 9 & 0 & 0 & 38 & 3 \\
\hline AAT loud reading & 29 & 26 & 26 & 30 & 28 & 27 & 29 \\
\hline AAT composing words & 28 & 28 & 29 & 30 & 30 & 29 & 24 \\
\hline AAT writing to dictation & 28 & 29 & 24 & 30 & 30 & 27 & 8 \\
\hline
\end{tabular}

CERAD = Consortium to Establish a Registry in Alzheimer's Disease - neuropsychological battery, BNT = Boston Naming Test, FTLD-CDR $=$ FTLD specific clinical dementia rating; MMSE $=$ Mini-Mental-State Exam, WMS = Wechsler Memory Scale, AAT Aachen Aphasia Test; * handedness was assessed with the Edinburgh Handedness Inventory (Oldfield, 1971) 
Table.2 Errors per lobe and hemisphere during object naming (patient $1-5$ ) and number naming (patient 6,7)
Error counts per hemisphere and lobe summed up to the overall error count in the last column

Patients \#2 an \#3 are forced right-handed

\begin{tabular}{llll}
\hline Error class & Errors left hemisphere & Errors right hemisphere & Total errors \\
\hline no response & $49(32.9 \%)$ & $76(37.2 \%)$ & $125(35.4 \%)$ \\
semantic & $21(14.1 \%)$ & $24(11.8 \%)$ & $45(12.8 \%)$ \\
performance & $18(12.1 \%)$ & $28(13.7 \%)$ & $46(13.0 \%)$ \\
timing & $61(40.9 \%)$ & $76(37.3 \%)$ & $137(38.8 \%)$ \\
total & $149(100 \%)$ & $204(100 \%)$ & $353(100 \%)$ \\
\hline
\end{tabular}

\begin{tabular}{|c|c|c|c|c|c|c|c|}
\hline Patient & Hemisphere & Frontal & Central & Temporal & Parietal & Sum hemisphere & Sum brain \\
\hline \multirow[t]{2}{*}{1} & left & 31 & 12 & 14 & 17 & 74 & 144 \\
\hline & right & 20 & 15 & 18 & 17 & 70 & \\
\hline \multirow[t]{2}{*}{2} & left & 3 & 1 & 0 & 0 & 4 & 11 \\
\hline & right & 3 & 3 & 0 & 1 & 7 & \\
\hline \multirow[t]{2}{*}{3} & left & 7 & 8 & 2 & 3 & 20 & 55 \\
\hline & right & 12 & 6 & 13 & 4 & 35 & \\
\hline \multirow[t]{2}{*}{4} & left & 4 & 0 & 1 & 0 & 5 & 11 \\
\hline & right & 4 & 1 & 1 & 0 & 6 & \\
\hline \multirow[t]{2}{*}{5} & left & 7 & 1 & 5 & 1 & 14 & 37 \\
\hline & right & 6 & 2 & 12 & 3 & 23 & \\
\hline \multirow[t]{2}{*}{6} & left & 4 & 6 & 4 & 5 & 19 & 64 \\
\hline & right & 7 & 17 & 9 & 12 & 45 & \\
\hline \multirow[t]{4}{*}{7} & left & 4 & 2 & 3 & 4 & 13 & 31 \\
\hline & right & 9 & 4 & 5 & 0 & 18 & \\
\hline & $\begin{array}{l}\text { Sum left } \\
\text { Sum right }\end{array}$ & & & & & $\begin{array}{l}149(42 \%) \\
204(58 \%)\end{array}$ & \\
\hline & Sum total & $121(34.3 \%)$ & $78(22.1 \%)$ & $87(24.6 \%)$ & $67(19.0 \%)$ & $353(100 \%)$ & \\
\hline
\end{tabular}

Table.3 Error classes per hemisphere

\section{Navigated rTMS}

Consortium to Establish a Registry in Alzheimer's Disease (CERAD) neuropsychological battery (Morris, 1993), which includes the Mini-Mental-State Examination (Folstein et al., 1975) and parts of the Aachener Aphasie test (Huber et al., 1983) (Table 1). Dementia severity was rated using the frontotemporal lobar degeneration-specific clinical dementia rating (FTLD-CDR) (Knopman et al., 2008). Neuropsychological assessment was performed within a maximum of eight weeks (median 13 days) before or after the nrTMS mappings.

In all patients cranial MRI was performed at 3 Tesla (Ingenia Elition X, Philips Healthcare, Best, The Netherlands). The protocol included a three-dimensional (3D) T1-weighted gradient echo sequence (repetition time/echo time: $9 / 4 \mathrm{~ms}, 1 \mathrm{~mm}^{3}$ isovoxel covering the whole head, no application of an intravenous contrast agent) for neuronavigation. Furthermore, ${ }^{18}$ F-FDG-PET was performed in all patients.

\section{Examination of naming performance}

Naming task parameters were adapted to each individual participant's performance prior to the nrTMS mappings. During two baseline sessions the patients had to name schematic black and white drawings of 80 common objects (one or two syllables), based on the Snodgrass and Vanderwart images (Snodgrass \& Vanderwart, 1980). The objects were presented on a screen one after another. After each of two naming sessions without stimulation (baseline sessions), the object was sorted out when the patient was not able to name the object promptly and correctly.

Two patients (\#6 and \#7) were not able to correctly name the majority of objects (errors in $84.4 \%$, and $59.7 \%$, respectively). Consequently, the nrTMS examination based on object naming seemed not feasible. However, both patients showed less difficulties in a number naming task (patient \#6: 
24\% errors; patient \#7: $36.1 \%$ errors). Therefore, the object naming task was replaced by a number (Figs. 0, 1, 2, 3, 4, 5, $6,7,8$ and 9) naming task in these two cases.

\section{Language mapping}

Language mapping by nrTMS was performed with the Nexstim eXimia 5.1 NBS system (Nexstim Plc., Helsinki, Finland) using a figure-of- 8 coil. The technical procedure and the protocol for language mapping has been described in detail previously (Picht et al., 2013; Tarapore et al., 2013; Krieg et al., 2014; Ille et al., 2016; Sollmann et al., 2017a, b; Krieg et al., 2013). In brief, cortical tissue was temporarily inhibited by a train of five repetitive nrTMS pulses at standardized stimulation targets in each hemisphere during a naming task. For neuronavigation, a co-registration of each patient's head and the according 3D MRI data was performed. Stimulation intensity was calibrated by the resting motor threshold (rMT) of hand muscles. The rMT is defined as lowest TMS intensity provoking a $50 \mu \mathrm{V}$ peak-topeak amplitude in the relaxed right first dorsal interosseus muscle in five out of ten stimulations (Rossini et al., 2015).

Forty-six standardized stimulation targets per hemisphere, distributed over 21 gyral segments, were set up on each patient's reconstructed head model within the nrTMS software according to a template based on a parcellation scheme described by Corina and colleagues (Corina et al., 2005; Tussis et al., 2016, 2017). The pre-selected objects (or numbers) were presented time-locked to nrTMS trains ( $5 \mathrm{~Hz}, 100 \%$ rMT). Keeping the coil perpendicular to the gyrus three nrTMS trains of five stimuli were applied at each target simultaneously with the picture presented on the screen. Thus, each of the targets was stimulated three times during the presentation of three pictures, leading to 276 stimulations per exam in each patient. The patients had to name the objects (or numbers) as quickly as possible. Then the stimulating coil was randomly moved to the next target during the inter-picture interval of $2.5 \mathrm{~s}$, and the picture-totrigger-interval was $0 \mathrm{~ms}$ with a display time of $700 \mathrm{~ms}$ for each picture (Krieg et al., 2014, 2016, 2017; Sollmann et al., 2014, 2017b; Tussis et al., 2016, 2017, 2020).

\section{Classification of naming errors}

In accordance with prior nTMS studies errors were classified into „no response“ (no, strongly distorted or incomplete utterance), ,semantic error“ (incorrect choice of word), „performance error" (correct choice of word, but utterance or assembly incorrect), and "timing error" (delay, hesitation) (Krieg et al., 2016; Lioumis et al., 2012; Picht et al., 2013; Sollmann et al., 2014, 2017b). The error classes "neologism" and "circumlocution" originally described as error classes by Corina and colleagues, were summarized as semantic errors. Phonological errors were subsumed among performance errors.

\section{Analysis}

For the detailed and standardized assessment - blinded to the sites of cortical stimulation - the baseline examination of the naming performance and the nrTMS examination with patients' answers were video-recorded (Picht et al., 2013; Sollmann et al., 2014). The videos recorded during nrTMS language mappings were analyzed, and any naming impairment was compared with the corresponding baseline response. Videos were rated in ELAN, an annotation tool for multi-media resources developed by the Max Planck Institute for Psycholinguistics that integrates tightly with Praat (version 6.1.53; Praat: doing phonetics by computer, http:// www.praat.org/) (Boersma \& van Heuven, 2001; Wittenburg et al., 2006). The spatial distribution of the naming errors that resulted from the virtual lesions revealed a map of cortical language areas. Additionally, the calculation of error rates was performed for each subject and each hemisphere by dividing the number of errors by the number of stimulations (per stimulated spot). Naming errors presumably due to pain or discomfort related to the stimulation were discarded.

\section{Results}

Seven patients with nfvPPA participated in the study. Patient characteristics and neuropsychological test results are displayed in Table 1. Clinical Gorno-Tempini criteria for nfvPPA were fulfilled in all patients. All participants showed a frontal and temporal atrophy in cranial MRI and a left frontal hypometabolism in ${ }^{18}$ F-FDG-PET. In five patients an amyloid PET was obtained that was negative in all cases. In the remaining two patients a lumbar puncture was performed: CSF levels of amyloid- $\beta 42$ were normal.

\section{Error counts}

As displayed in Table 2 a total of 353 naming errors during 1932 stimulations was observed in nfvPPA patients, which amounts to an error rate of $18.2 \%$. The majority of errors occurred during stimulation of the frontal lobes (34.3\%), followed by the temporal lobes $(24.6 \%)$ and the central lobes $(22.1 \%)$.

Error classes and distribution of errors in both hemispheres are shown in Table 3; Fig. 1. Overall, more errors were induced in the right hemisphere (204/353 errors, $58 \%)$ than in the left hemisphere (149/353 errors, $42 \%)$. Timing errors and non-responses were most frequent (39\% and $35 \%$ of all errors). 


\section{template}

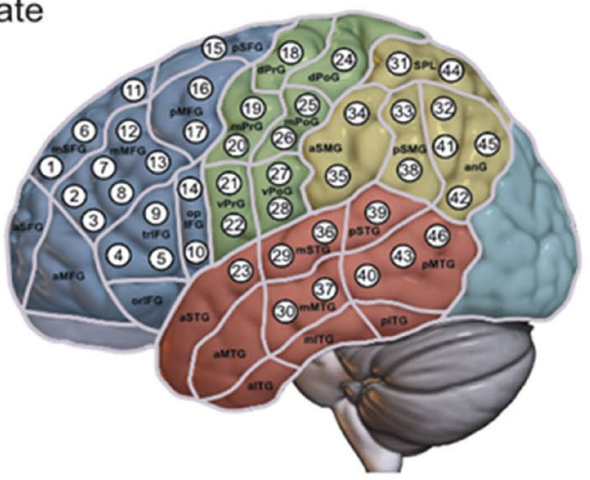

1 left

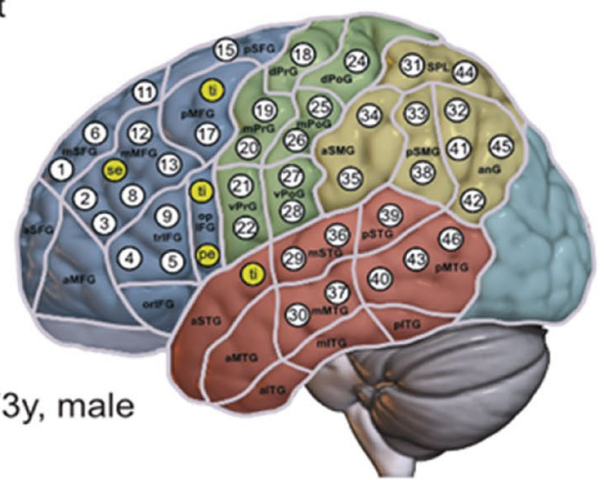

2

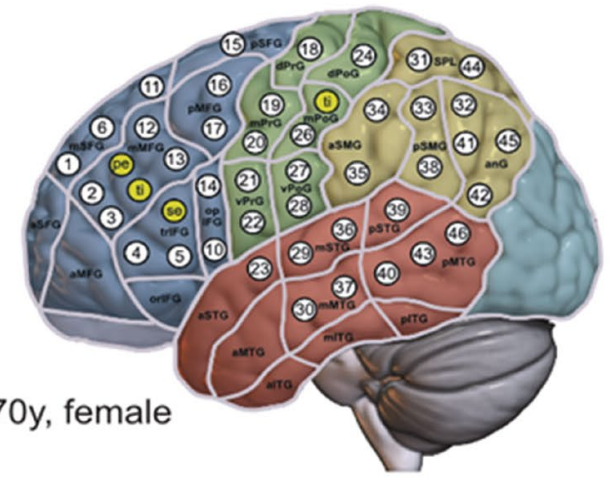

3

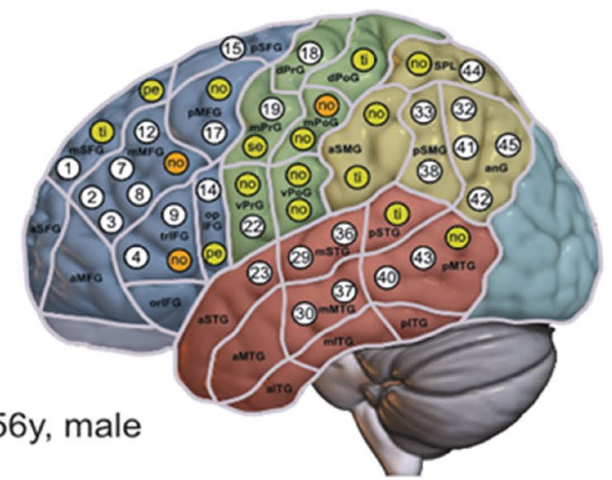

Fig. 1 Language maps - For all patients (1-7) nrTMS language-mappings of the left hemisphere (left column) and right hemisphere (right column) are shown. At each of the 46 standardized stimulation targets in every hemisphere a color code indicates the error count (white $=$ no error, yellow $=1$ error, orange $=2$ errors, red $=3$ errors) while a 2-letter code discloses the error class ranking highest among the errors at the target. A slightly modified parcellation scheme accord-

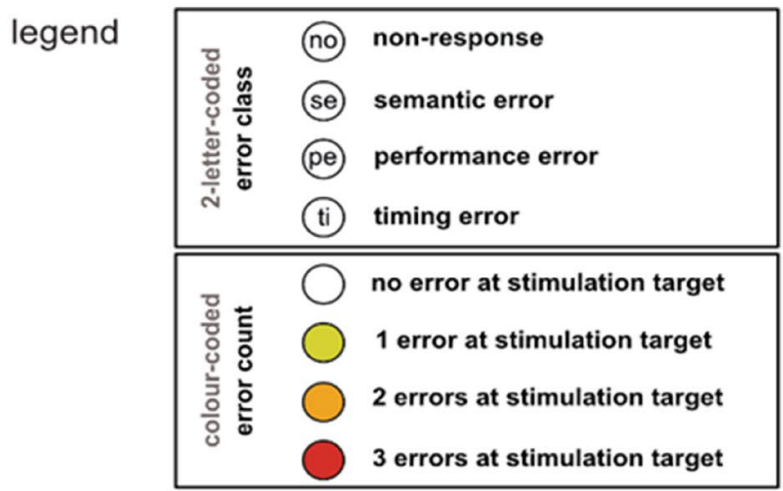

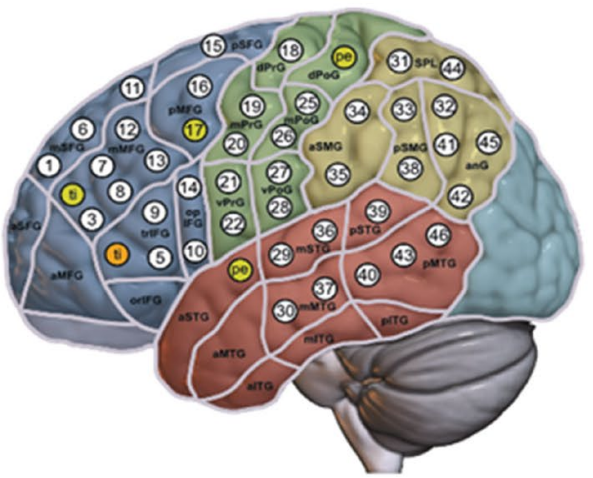

right
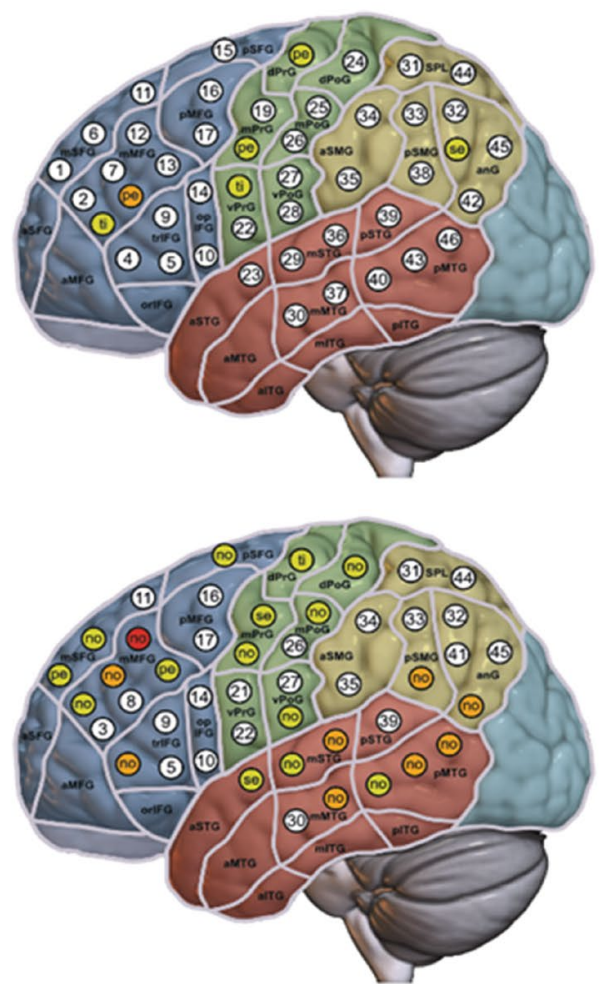

ing to Corina et al. is displayed on top of the MNI 2009b asymmetric template. Regions belonging to one lobe share the same color: blue in the frontal lobe, green in the central lobe, brown in the temporal lobe, ocher in the parietal lobe, and turquoise in the occipital lobe

The right hemisphere is projected into a template of the left hemisphere in order to facilitate comparability 
4 left

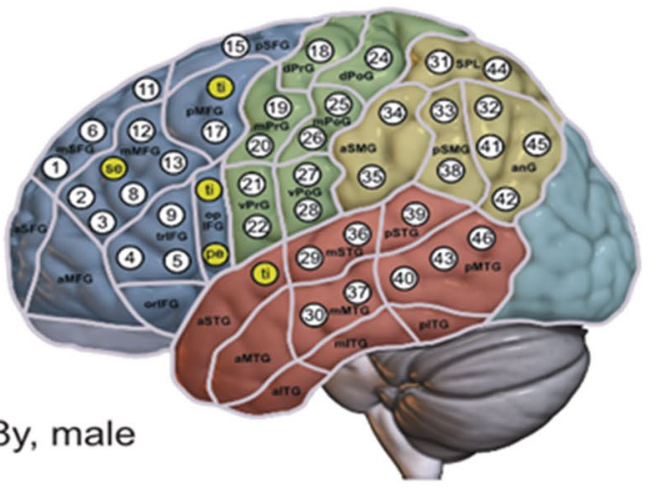

5

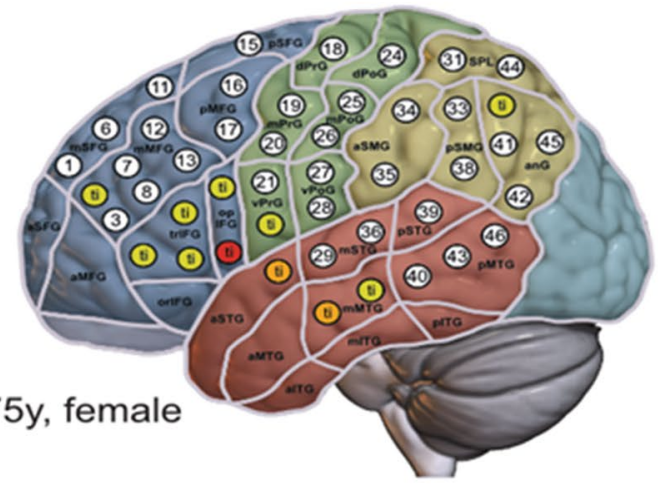

6

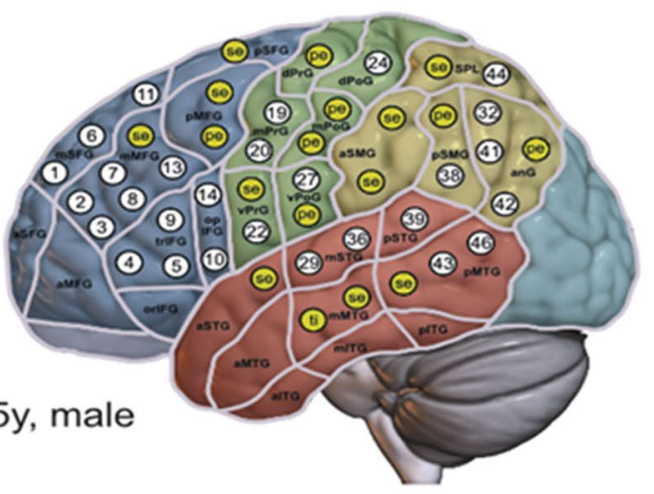

7

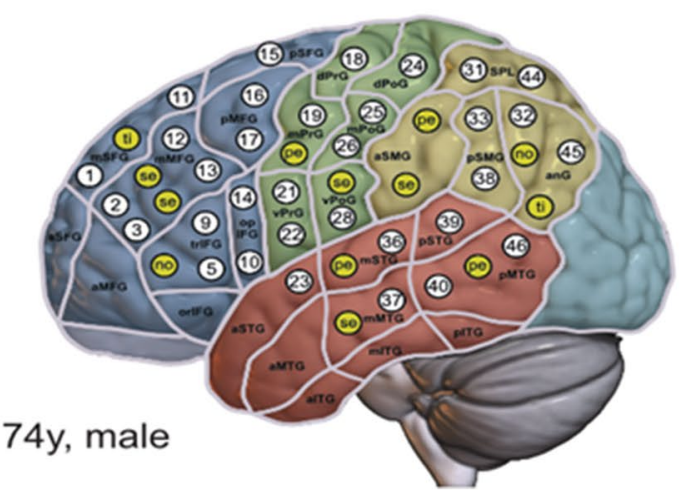

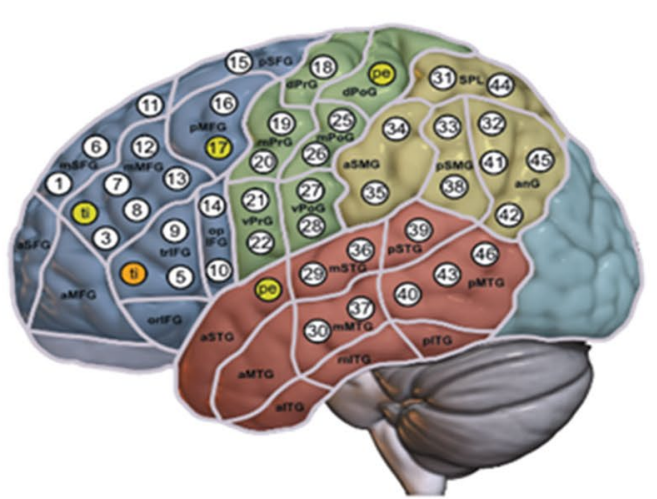

right
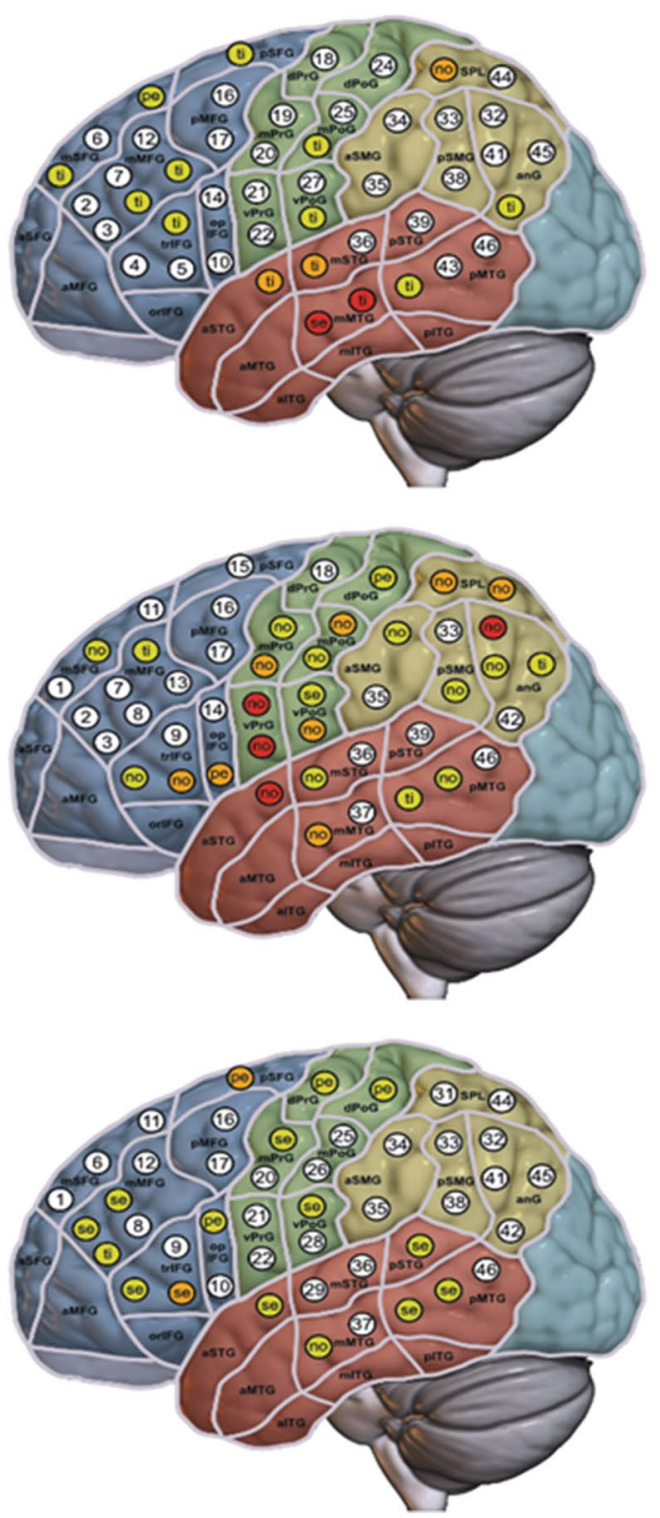

Fig. 1 (continued) 
Table.4 Discomfort and pain patient estimation on a 10-point Likert scale $(0=$ no discomfort/ pain)

\begin{tabular}{llllllll}
\hline Patient & $\# 1$ & $\# 2$ & $\# 3$ & $\# 4$ & $\# 5$ & $\# 6$ & $\# 7$ \\
\hline pain left hemisphere & 5 & 7 & 2 & 0 & 5 & 1 & 0 \\
pain right hemisphere & 6 & 5 & 3 & 0 & 5 & 1 & 8 \\
discomfort left hemisphere & 6 & 4 & 2 & 0 & 5 & 0 & 0 \\
discomfort right hemisphere & 7 & 3 & 3 & 0 & 5 & 0 & 0 \\
\hline
\end{tabular}

\section{Tolerability}

Overall, the nrTMS examination was tolerated well by six out of seven patients. All of the patients stated after the examination that they would be willing to undergo further nrTMS sessions. However, the majority of patients disclosed considerable discomfort and pain (Table 4). As observed in the video recordings and confirmed by the participants, pain and discomfort were limited to stimulation of frontal regions.

\section{Discussion}

To our knowledge the present study is the first that performed language mapping in neurodegenerative aphasia using nrTMS. Specifically, nrTMS induced an overall error rate of $18 \%$ in nfvPPA patients. Previous studies using nrTMS in healthy individuals have described similar error rates (Krieg et al., 2016; Sollmann et al., 2014, 2017b). Timing errors and no responses were most frequent in nfvPPA patients (39\% and 35\% of all errors), in line with the fact that halting speech is one of the two core features of nvfPPA (Gorno-Tempini et al., 2011).

Although we observed an obvious inter-individual variability regarding error rates and language maps, stimulation of the temporal, central, and parietal lobes induced fewer errors than stimulation of frontal areas. This goes along with the fact that nfvPPA is characterized by imaging abnormalities in the posterior fronto-insular region (Gorno-Tempini et al., 2011). Specifically, the middle middle frontal gyrus (mMFG) was identified as a region with prevalent errors in both hemispheres, which seems to extend to the posterior MFG (pMFG) in several cases. Cortical thickness in the MFG of both hemispheres was shown to correlate with reduced fluency in PPA (Rogalski et al., 2011). Furthermore, the MFG is also a spot of nfvPPA-typical hypometabolism in FDG-PET (Routier et al., 2018). In a recent tDCS treatment trial, individuals with reduced left MFG volumes benefited more from tDSC than those receiving sham (de Aguiar et al., 2020). Other regions with prevalent errors are the triangular inferior frontal gyrus (trIFG) and opercular IFG (opIFG), which mostly overlap with Broca's area in the left hemisphere. While this replicates the importance of Broca's area for human language in general, language function in nfvPPA is by no means confined to these regions, but widespread. In five out of seven patients (all except patients \#4 and \#5) nTMS language maps point towards a particular role of the central gyri in nfvPPA language production. This is consistent with findings in stroke patients who showed involvement of the precentral gyrus in apraxia of speech (Itabashi et al., 2016).

Surprisingly, more errors were induced in the right hemisphere $(58 \%)$ than in the left hemisphere (42\%). A shift of language function towards the right or unaffected hemispheres has already been suggested in stroke, epilepsy, and intracranial neoplasms (Anglade et al., 2014; Baciu \& Perrone-Bertolotti, 2015; Ille et al., 2016; Krieg et al., 2013). There is broad consensus that this phenomenon indicates cortical reorganization. Despite some evidence from functional imaging for a language shift in nfvPPA patients (Vandenbulcke et al., 2005), the general understanding so far is that patients with nfvPPA subtypes are not remapping language functions to contralateral networks. They appear to use pre-existing language areas, but with progressively declining efficiency (Norise \& Hamilton, 2017). Notably, these assumptions are based on comparatively weak evidence (Wilson et al., 2010). Yet, cortical reorganization in nfvPPA and other neurodegenerative disorders fuels hope that nrTMS might become a treatment option, either by stimulation of compensatory right hemispheric networks or by stimulation of pathologically weakened left hemispheric networks in order to enhance the remaining language function (Norise \& Hamilton, 2017).

However, an alternative explanation for the distributions of error rates in each hemisphere could also directly relate to degenerative processes: a relative increase in the number of errors following stimulation could depend on the high degree of impairment of neural plasticity in the left hemisphere, which is the main focus of the neurodegenerative process in PPA. Further studies that combine nrTMS with functional or metabolic imaging to specifically stimulate circumscribed areas with altered activity profiles may help to shed light on the distinct causes for the observed error distributions between hemispheres.

The present study has some obvious limitations. First, the sample size is small and the patient sample was not homogenous regarding handedness. Second, not all patients were able to perform an object naming task so that a number naming task was used in two patients. Third, a general obstacle of language 
mapping with nrTMS is that stimulation targets at the edge of the frontal lobe are not included since stimulation at these targets causes intolerable discomfort in many patients. Consequently, the polar to orbital parts of the frontal lobe are not covered. In addition, since naming errors that were caused by discomfort or pain were discarded from the analysis, the error rate in frontal regions was potentially underestimated. Finally, the study lacks healthy control probands that would allow a comparison of the performance.

However, the present study is the first to show that language mapping in neurodegenerative aphasia using nrTMS is feasible and offers unique insights into language function by combining good temporary with good spatial resolution. Evaluating the individual language function might be a prerequisite for any targeted, personalized stimulation therapy. The elevated right-hemispheric error rate in our study may be indicative of a partial shift of language function to the right hemisphere in nfvPPA patients during the course of disease and therefore might point to the existence of neuronal plasticity in neurodegenerative aphasia. Further, longitudinal studies are needed including larger patient samples and healthy controls to further investigate language function and brain plasticity in nvfPPA. In such larger cohort studies, our current data justifies investigating nrTMS error distribution as a new neurophysiological biomarker especially for the early phase of nfvPPA.

\section{Conclusions}

Our pilot study showed that nrTMS is a feasible tool for language mapping in nfvPPA. Evaluating the individual language function might be a prerequisite for any targeted, personalized stimulation therapies. Study results suggests the existence of neuronal plasticity in neurodegenerative aphasia.

Acknowledgements We would like to thank all patients for their invaluable contribution to this project. Further, we would like to thank the bioinformatiX.org research group of the Munich Medical Society for supporting this trial by providing access to its technical infrastructure.

Author contribution Conception and study design (JDS, FMS, SK, NS, SI), data collection and acquisition (FMS, AS, LH, JDS), analyses (FMS, LH, TG), interpretation of results (FMS, JDS, NS, SI, TG, SK), drafting the manuscript (FMS, JDS), revising the manuscript critically for important intellectual content (JDS, NS, SI, TG, SK), approval of final version (all authors).

Funding Open Access funding enabled and organized by Projekt DEAL.

Data availability The data that support the findings of this study are available from the corresponding author, upon reasonable request. The data are not publicly available due to ethical and legal restrictions. Due to German data protection rules transfer of data that is considered to be personal by local laws (for example video and sound recordings) is forbidden. For the same legal reasons transmission of data might be possible within the European Union only.

\section{Declarations}

Ethical approval The study was approved by the local ethics committee (\#377/18 S) and conducted in line with the principles expressed in the Declaration of Helsinki.

Consent to participate Each participant provided written informed consent prior to enrollment.

Consent to publish Not applicable.

Conflict of interest Felix Müller-Sarnowski, Timo Grimmer and Janine Diehl-Schmid have no competing interests with regard to the submitted manuscript. Outside the submitted manuscript TG received consulting fees from Abbvie, Anavex, Biogen, Bracket, Eli Lilly, Functional Neuromodulation, Iqvia/Quintiles, Novartis, Novo Nordisk, NuiCare, Roche Pharma, Toyama, and Vivoryon; lecture fees from Actelion, B.Braun, Biogen, Eli Lilly, Life Molecular Imaging, Novartis, Parexel, and Roche Pharma; and grants to his institution from Actelion and Novartis. Sandro Krieg is consultant for Ulrich Medical (Ulm, Germany) and received honoraria from Nexstim Plc (Helsinki, Finland), Spineart Deutschland GmbH (Frankfurt, Germany), Medtronic (Meerbusch, Germany) and Carl Zeiss Meditec (Oberkochen, Germany). Sandro Krieg and Sebastian Ille are consultants for Brainlab AG (Munich, Germany). Sebastian Ille and Nico Sollmann received honoraria from Nexstim Plc (Helsinki, Finland).

Open Access This article is licensed under a Creative Commons Attribution 4.0 International License, which permits use, sharing, adaptation, distribution and reproduction in any medium or format, as long as you give appropriate credit to the original author(s) and the source, provide a link to the Creative Commons licence, and indicate if changes were made. The images or other third party material in this article are included in the article's Creative Commons licence, unless indicated otherwise in a credit line to the material. If material is not included in the article's Creative Commons licence and your intended use is not permitted by statutory regulation or exceeds the permitted use, you will need to obtain permission directly from the copyright holder. To view a copy of this licence, visit http://creativecommons.org/licenses/by/4.0/.

\section{References}

Anglade, C., Thiel, A., \& Ansaldo, A. I. (2014). The complementary role of the cerebral hemispheres in recovery from aphasia after stroke: A critical review of literature. Brain Injury, 28(2), $138-145$

Baciu, M., \& Perrone-Bertolotti, M. (2015). What do patients with epilepsy tell us about language dynamics? A review of fMRI studies. Reviews in the Neurosciences, 26(3), 323-341

Boersma, P., \& van Heuven, V. (2001). Speak and unSpeak with PRAAT. Glot International, 5(9/10), 341-347

Corina, D. P., Gibson, E. K., Martin, R., Poliakov, A., Brinkley, J., \& Ojemann, G. A. (2005). Dissociation of action and object naming: Evidence from cortical stimulation mapping. Human Brain Mapping, 24(1), 1-10

Cotelli, M., Manenti, R., Alberici, A., Brambilla, M., Cosseddu, M., Zanetti, O., \& Borroni, B. (2012). Prefrontal cortex rTMS enhances action naming in progressive non-fluent aphasia. European Journal of Neurology, 19(11), 1404-1412 
Cotelli, M., Manenti, R., Ferrari, C., Gobbi, E., Macis, A., \& Cappa, S. F. (2020). Effectiveness of language training and non-invasive brain stimulation on oral and written naming performance in Primary Progressive Aphasia: A meta-analysis and systematic review. Neuroscience \& Biobehavioral Reviews, 108, 498-525

de Aguiar, V., Zhao, Y., Faria, A., Ficek, B., Webster, K. T., Wendt, H., ... Tsapkini, K. (2020). Brain volumes as predictors of tDCS effects in primary progressive aphasia. Brain and Language, 200, 104707

Folstein, M. F., Folstein, S. E., \& McHugh, P. R. (1975). "Mini-mental state": A practical method for grading the cognitive state of patients for the clinician. Journal of Psychiatric Research, 12(3), 189-198

Gorno-Tempini, M. L., Hillis, A. E., Weintraub, S., Kertesz, A., Mendez, M., Cappa, S. F. ... Grossman, M. (2011). Classification of primary progressive aphasia and its variants. Neurology, 76(11), 1006-1014

Huber, W., Poeck K., Weniger, D., Wilmes, K. (1983). Aachener Aphasie Test. Hogrefe Verlag, Göttingen, Zürich

Ille, S., Kulchytska, N., Sollmann, N., Wittig, R., Beurskens, E., Butenschoen, V. M. ... Krieg, S. M. (2016). Hemispheric language dominance measured by repetitive navigated transcranial magnetic stimulation and postoperative course of language function in brain tumor patients. Neuropsychologia, 91, 50-60

Itabashi, R., Nishio, Y., Kataoka, Y., Yazawa, Y., Furui, E., Matsuda, M., \& Mori, E. (2016). Damage to the Left Precentral Gyrus Is Associated With Apraxia of Speech in Acute Stroke. Stroke, 47(1), 31-36

Knopman, D. S., Kramer, J. H., Boeve, B. F., Caselli, R. J., Graff-Radford, N. R., Mendez, M. F. ... Mercaldo, N. (2008). Development of methodology for conducting clinical trials in frontotemporal lobar degeneration. Brain, 131(11), 2957-2968

Krieg, S. M., Lioumis, P., Mäkelä, J. P., Wilenius, J., Karhu, J., Hannula, H. ... Picht, T. (2017). Protocol for motor and language mapping by navigated TMS in patients and healthy volunteers; workshop report. Acta Neurochirurgica, 159(7), 1187-1195

Krieg, S. M., Sollmann, N., Hauck, T., Ille, S., Foerschler, A., Meyer, B., \& Ringel, F. (2013). Functional language shift to the right hemisphere in patients with language-eloquent brain tumors. PLoS ONE, 8(9), e75403

Krieg, S. M., Sollmann, N., Tanigawa, N., Foerschler, A., Meyer, B., \& Ringel, F. (2016). Cortical distribution of speech and language errors investigated by visual object naming and navigated transcranial magnetic stimulation. Brain Structure and Function, 221(4), 2259-2286

Krieg, S. M., Tarapore, P. E., Picht, T., Tanigawa, N., Houde, J., Sollmann, N., ... Nagarajan, S. (2014). Optimal timing of pulse onset for language mapping with navigated repetitive transcranial magnetic stimulation. NeuroImage, 100, 219-236

Lioumis, P., Zhdanov, A., Mäkelä, N., Lehtinen, H., Wilenius, J., Neuvonen, T. ... Mäkelä, J. P. (2012). A novel approach for documenting naming errors induced by navigated transcranial magnetic stimulation. Journal of Neuroscience Methods, 204(2), 349-354

Morris, J. C. (1993). The Clinical Dementia Rating (CDR): Current version and scoring rules. Neurology, 43(11), 2412-2414

Norise, C., \& Hamilton, R. H. (2017). Non-invasive brain stimulation in the treatment of post-stroke and neurodegenerative aphasia: parallels, differences, and lessons learned. Frontiers in Human Neuroscience, 10. https://doi.org/10.3389/fnhum.2016.00675

Oldfield, R. C. (1971). The assessment and analysis of handedness: The Edinburgh inventory. Neuropsychologia, 9, 97-113.

Picht, T., Krieg, S. M., Sollmann, N., Rösler, J., Niraula, B., Neuvonen, T., ... Ringel, F. (2013). A Comparison of Language Mapping by Preoperative Navigated Transcranial Magnetic Stimulation and Direct Cortical Stimulation During Awake Surgery. Neurosurgery, 72(5), 808-819
Rogalski, E., Cobia, D., Harrison, T. M., Wieneke, C., Thompson, C. K., Weintraub, S., \& Mesulam, M. M. (2011). Anatomy of Language Impairments in Primary Progressive Aphasia. The Journal of Neuroscience, 31(9), 3344-3350

Rossini, P. M., Burke, D., Chen, R., Cohen, L. G., Daskalakis, Z., Di Iorio, R. ... Ziemann, U. (2015). Non-invasive electrical and magnetic stimulation of the brain, spinal cord, roots and peripheral nerves: Basic principles and procedures for routine clinical and research application. An updated report from an I.F.C.N. Committee. Clinical Neurophysiology: Official Journal of the International Federation of Clinical Neurophysiology, 126(6), 1071-1107

Routier, A., Habert, M. O., Bertrand, A., Kas, A., Sundqvist, M., Mertz, J. ... Teichmann, M. (2018). Structural, microstructural, and metabolic alterations in primary progressive aphasia variants. Frontiers in Neurology, 9. https://doi.org/10.3389/fneur. 2018.00766

Snodgrass, J. G., \& Vanderwart, M. (1980). A standardized set of 260 pictures: Norms for name agreement, image agreement, familiarity, and visual complexity. Journal of Experimental Psychology. Human Learning and Memory, 6(2), 174-215

Sollmann, N., Ille, S., Negwer, C., Boeckh-Behrens, T., Ringel, F., Meyer, B., \& Krieg, S. M. (2017a). Cortical time course of object naming investigated by repetitive navigated transcranial magnetic stimulation. Brain Imaging and Behavior, 11(4), 1192-1206

Sollmann, N., Meyer, B., \& Krieg, S. M. (2017b). Implementing functional preoperative mapping in the clinical routine of a neurosurgical department: technical note. World Neurosurgery, 103, 94-105

Sollmann, N., Tanigawa, N., Ringel, F., Zimmer, C., Meyer, B., \& Krieg, S. M. (2014). Language and its right-hemispheric distribution in healthy brains: An investigation by repetitive transcranial magnetic stimulation. NeuroImage, 102(Pt 2), 776-788

Tarapore, P. E., Findlay, A. M., Honma, S. M., Mizuiri, D., Houde, J. F., Berger, M. S., \& Nagarajan, S. S. (2013). Language mapping with navigated repetitive TMS: Proof of technique and validation. NeuroImage, 82, 260-272

Tussis, L., Sollmann, N., Boeckh-Behrens, T., Meyer, B., \& Krieg, S. M. (2016). Language function distribution in left-handers: A navigated transcranial magnetic stimulation study. Neuropsychologia, 82, 65-73

Tussis, L., Sollmann, N., Boeckh-Behrens, T., Meyer, B., \& Krieg, S. M. (2017). Identifying cortical first and second language sites via navigated transcranial magnetic stimulation of the left hemisphere in bilinguals. Brain and Language, 168, 106-116

Tussis, L., Sollmann, N., Boeckh-Behrens, T., Meyer, B., \& Krieg, S. M. (2020). The cortical distribution of first and second language in the right hemisphere of bilinguals - an exploratory study by repetitive navigated transcranial magnetic stimulation. Brain Imaging and Behavior, 14(4), 1034-1049

Vandenbulcke, M., Peeters, R., Van Hecke, P., \& Vandenberghe, R. (2005). Anterior temporal laterality in primary progressive aphasia shifts to the right. Annals of Neurology, 58(3), 362-370

Wilson, S. M., Henry, M. L., Besbris, M., Ogar, J. M., Dronkers, N. F., Jarrold, W. ... Gorno-Tempini, M. L. (2010). Connected speech production in three variants of primary progressive aphasia. Brain, 133(7), 2069-2088

Wittenburg, P., Brugman, H., Russel, A., Klassmann, A., \& Sloetjes, H. (2006). ELAN: a Professional Framework for Multimodality Research. Proceedings of LREC 2006, Fifth International Conference on Language Resources and Evaluation, 4

Publisher's note Springer Nature remains neutral with regard to jurisdictional claims in published maps and institutional affiliations. 